\title{
Antifouling PVDF membrane by surface covalently anchoring functionalized graphene quantum dots
}

\author{
Wenyao Shao ${ }^{\mathrm{b}}$, Hanjun Ma a,b, Tong Yu a,d, Chenpu Wu a,b, Zhuan Hong a,d, Ying Xiong c, \\ Quanling Xie ${ }^{\mathrm{a}, \mathrm{d}, *}$

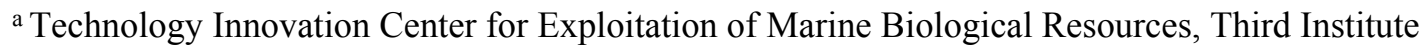 \\ of Oceanography, Ministry of Natural Resources, Xiamen 361005, PR China \\ ${ }^{b}$ Department of Chemical and Biochemical Engineering, College of Chemistry and Chemical \\ Engineering, Xiamen University, Xiamen 361005, PR China \\ ${ }^{c}$ Guangdong Provincial Key Laboratory of Soil and Groundwater Pollution Control, School of \\ Environmental Science and Engineering, Southern University of Science and Technology, \\ Shenzhen 518055, PR China \\ ${ }^{\mathrm{d}}$ Fujian Collaborative Innovation Center for Exploitation and Utilization of Marine Biological \\ Resources, Xiamen, 361005, PR China
}

\footnotetext{
* Corresponding author.

E-mail address: qlxie@tio.org.cn (Q. Xie)
} 


$$
\mathrm{H}_{2} \mathrm{~N} \smile \mathrm{NH}_{2}
$$

(a)

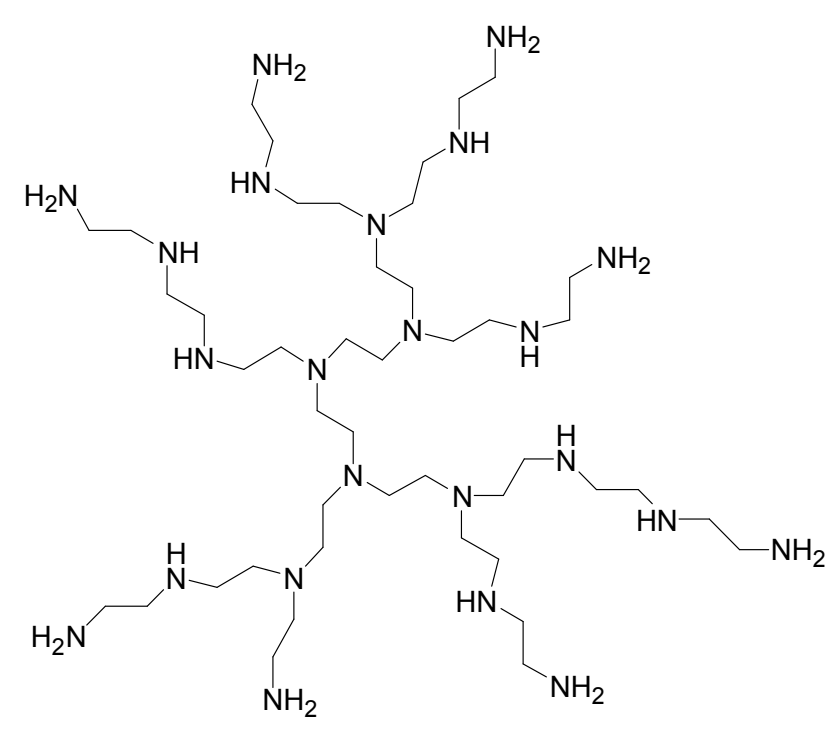

(b)

Fig. S1. The molecular structures of EDA (a) and HPEI (b)

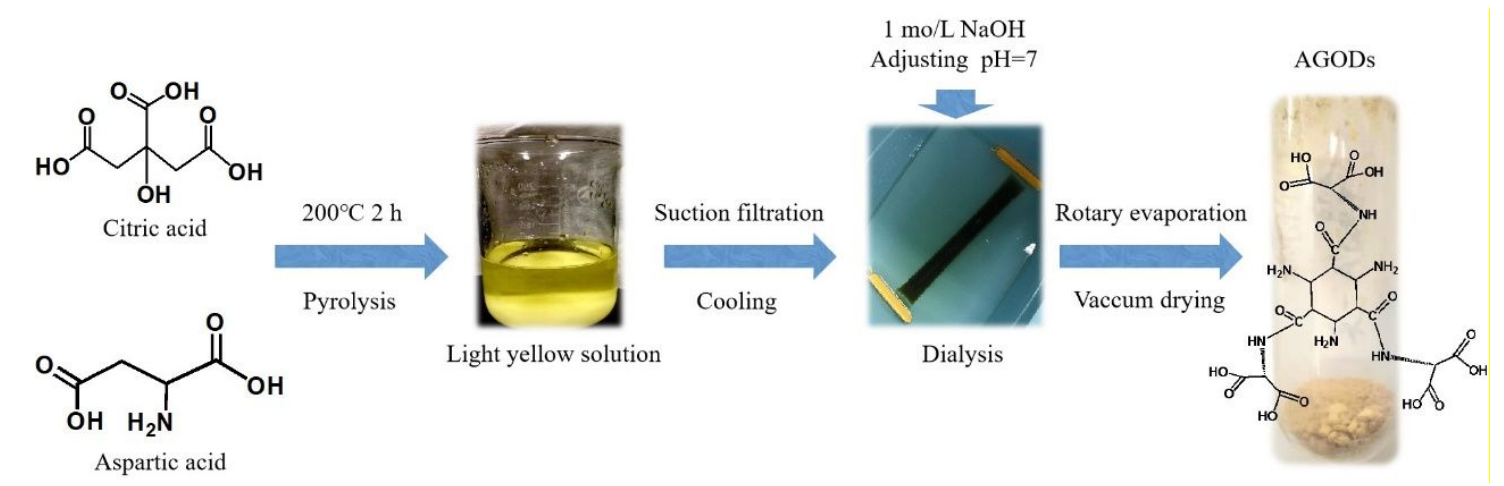

Fig. S2. Synthesis procedures of AGQD. 